\section{Military Technical College Kobry El-kobbah, Cairo, Egypt}

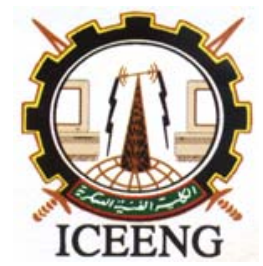

\author{
$5^{\text {th }}$ International Conference \\ on Electrical Engineering \\ ICEENG 2006
}

\title{
MULTIOBJECTIVE OPTIMISATION OF SWITCHED RELUCTANCE MOTOR USING FUZZY-GENETIC-SIMPLEX ALGORITHM
}

\author{
Dr. Amged El-Wakeel ${ }^{*}$
}

\begin{abstract}
This paper presents a new method for multiobjective optimisation of a switched reluctance motor. Four objective functions regarding motor efficiency, power factor, torque ripples and outer volume are considered. The proposed method combines fuzzy logic, genetic algorithm and simplex technique as a general global optimisation technique. The new technique is searching for the best compromise solution, which maximises the designer total degree of satisfaction. In order to predict the motor performance accurately , a hybrid FEA-analytical simulation model has been adopted. The model combines some of the FEA accuracy with the simplicity of analytical model. A full time stepping FEA analysis for the optimised motor has been done to verify the final design of the motor.
\end{abstract}

\section{KEY WORDS}

Switched Reluctance Motors, Fuzzy Logic, Genetic Algorithms, Optimisation Methods, Finite Element Analysis.

\section{INTRODUCTON}

Many real world electromagnetic problems involve simultaneous optimisation of multiple objectives that are usually in conflict such as volume and efficiency. Generally there are two ways to solve such optimisation problems. One way is to get the best compromise solution and the other way is to get all non-inferior (Pareto) solutions. In compromise solution methods, the original multiobjective problem (MOP) is converted to a single-objective problem (SOP) and then solved by any of the deterministic techniques. From this category weighted sum method in which the multiobjective optimisation problem is converted to a single-objective optimisation problem by weighing the objectives with a weighting vector. Weights are usually assigned according to the importance of objectives; more important objectives will get higher weights, and less important objectives will get lower weights. However, since not all objectives have the same range of values, they must also be normalised [1, 2]. The main disadvantages of this method are: the difficulty in choosing the best set of weights for the problem, and the difficulty of dealing with different quantities, which are measured in different scales [2]. In addition to the above drawbacks, the use of any well-known deterministic algorithm usually leads to a local optimum rather than a global optimum solution.

* Assistant professor, Electrical Power and Energy Department, MTC, Cairo, Egypt 
The other conventional way is to use constraint method. The constraint method involves optimising the main or primary objective function and expressing the other objectives in the form of constraints [1, 3]. This method has the advantage of converting problem to a simple single-objective problem. However, the method suffers from the difficulty of selecting suitable values of constraints to ensure a feasible solution[4].Too hard constraints may lead to no solution and at least one of the bounds must be released to solve the problem [1].

On the other hand, the concept of Pareto optimum was formulated by Pareto in 1896, it is considered as an essential searching part in this area [1]. In Pareto optimum, a solution can be superior, inferior, equal, and also indifferent to another solution with respect to another objective values. "Superior" means a solution that is not inferior in any objective and at least superior in one objective than another; the superior solution is also said to dominate the inferior one. Using this concept, one can define what an optimal solution is:” a solution which is not inferior to any other solution in the search space". Such a solution is called Pareto Optimal and the entire set of optimal trade-offs is called Pareto front or Pareto optimal set [5].

The main advantages of Pareto Optimum are that it takes all objectives into account simultaneously, and every element of Pareto front can be considered as a good solution[2]. However, the method is computationally expensive since it needs to maintain the front, and hence needs to store and continually update a large set of solutions. Also, it is not very good and intuitive if the number of objectives is large. In some cases, when all objectives are equally important, there are an incredible number of Pareto optimal solutions, which complicate the optimisation process.

\section{OPTIMISATION ALGORITHMS OF ELECTRICAL MACHINES}

In the design optimisation of electrical machines, the following issues are worth considering:

- There are usually many conflicting design objectives, which need to be optimised together.

- Many of conflicting objectives are measured in different scales (e.g. volume and efficiency).

- Most design procedures contain relaxable or soft constraints that can easily be represented by fuzzy sets, in addition to tight, or hard constraints.

- Sufficient information is usually available for the designer to determine his/her goals and hence the computationally expensive Pareto methods are not needed.

- Some expensive time methods such as sequential optimisation method are not justified for a real compromise solution.

From these issues it is clear that the constrained multiobjective optimisation formulation is the natural choice in electrical machine design optimisation.

Optimisation algorithms on the other hand, can be classified according to determinism into two categories: stochastic and Deterministic. Genetic algorithm is an example of stochastic techniques while simplex algorithm is an example of deterministic techniques. In this section, the two algorithms in addition to the new Fuzzy-genetic-simplex algorithm are explained.

\subsection{Genetic Algorithm}

Genetic algorithm has been applied to many electric machines [3, 6-8] but it still has not been tuned to get red of its inherent convergence slowness especially for large search space optimisation problems. The Genetic Algorithm can be summarised as follows [3]:

i) Build a fitness function from the objective function and constraints. 
ii) Create an initial population (usually randomly generated strings). The population consists of $\boldsymbol{N}$ strings or chromosomes, which in turn are composed of sub-strings or genes describing the design variables.

iii) Evaluate all of the individuals according to the fitness function.

iv) Select a new population from the old population based on the fitness of the individuals.

v) Apply genetic operators (mutation \& crossover) to members of the population to create new solutions.

vi) Evaluate these newly created individuals.

vii) Repeat steps from (iv) to (vii) until the termination criteria has been satisfied.

\subsection{Simplex Algorithm}

Simplex algorithm has also been applied to many electric machines [3, 9, 10]. Its main disadvantages are that the algorithm efficiency depends on starting point and the search step length. Also, the algorithm in general searches a point of local optimum unless the objective function is uni-modal. However the algorithm is very fast compared to genetic algorithm and does not depend on the accuracy of descent direction evaluation required by other conventional deterministic algorithms.

The Simplex algorithm is based on the idea of comparing the values of the objective functions at the $\mathrm{N}+1$ vertices of a polytope (Simplex) in N-dimensional space and moving the polytope towards the minimum point as the optimisation progress. The movement of the polytope towards the minimum point is achieved through three operations: Reflection, Contraction, and Expansion [11].

The algorithm can be outlined as follows:

i) Select a starting point.

ii) Construct a starting polytope consisting of the starting point and the additional points (one point in each dimension).

iii) Evaluate the objective function at each point.

iv) Replace the worst point by a new point using simplex operators (Reflection, Contraction, and Expansion)

vi) Repeat steps from (iii) to (vi) until the termination criteria has been satisfied.

\subsection{Genetic-Simplex Algorithm}

The genetic-simplex algorithm can be summarised as follows[12]:

i) Apply genetic algorithm to locate the interval, which likely contains the global minimum.

ii) Switch to the simplex method assuming the final solution of the genetic algorithm as a starting solution for simplex.

This technique combines a Genetic algorithm with the Simplex method and is aimed at solving the following problems [9, 11]:

- The genetic algorithm avoids local minima.

- The simplex algorithm avoids the problem of slowness and near-optimum convergence.

\subsection{Fuzzy-Genetic-Simplex (FGS) Algorithm}

This proposed algorithm has the following steps:

i) Aggregate the different in-conflict objectives in one objective using Fuzzy combination algorithm.

ii) Apply genetic algorithm to locate the interval, which likely contains the global minimum. 
iii) Switch to the simplex method assuming the final solution of the genetic algorithm as a starting solution for simplex.

This new algorithm is proposed to solve the following problems.

- The problem of decision-making coincides with weighted sum method by employing the fuzzy combination algorithm.

- The problem of too hard constraints coincides with constraint method by using fuzzy constraints.

- The problem of local minimum sticking by using genetic algorithm.

- The problem of slowness and near-optimum convergence by simplex algorithm[10, 13].

The proposed technique will be applied to optimise a Switched reluctance Motor for vehicle applications.

\section{PROBLEM FORMULATION}

This section describes the use of genetic and hybrid algorithms using real rather than binary encoding to obtain new designs for multiobjective optimisation problem that translates into significant cost and performance benefits for the switched reluctance motor. The switched reluctance motor has been selected because it has many advantages such as simple and robust construction with high reliability and low cost. The problem formulation includes the formulation of objective function, motor modelling, optimisation constraints and fitness function

\subsection{Objective Function and Optimisation Vector}

The objective function is selected to minimise the outer volume $\left(V_{\text {out }}\right)$ and Torque ripples $(T R)$ and maximise the power factor $(P F)$ and efficiency $(\eta)$ as:

$$
F(x)=\left\{V_{\text {out }} \quad P F \quad \eta \quad T R\right\}
$$

- $\quad$ The outer Ivolume can be expressed as [3]:

$$
V_{\text {out }}=\frac{\pi}{4}\left[\frac{D}{s r}\right]^{2} L_{e}
$$

where $D$ is the rotor outer diameter, $s r$ is the split ratio of rotor outer diameter to motor outer diameter and $L_{e}$ is the envelope length which is a function of stack length $l$ and diameter $D$ [3].

- The maximum power factor can be estimated as the ratio between output energy $(W)$ and the input energy $(W+R)$ in each working stroke [14]:

$$
P F=\frac{W}{W+R}
$$

- The per unit efficiency of motor can be computed as:

$$
\eta=\frac{P_{r}}{P_{r}+\Delta P_{c u}+\Delta P_{c}+\Delta P_{m}}
$$

where $P_{r}$ is the rated output power, $\Delta P_{c u}$ is the full load copper loss, $\Delta P_{c}$ is the core losses, and $\Delta P_{m}$ is the mechanical losses.

- Torque ripples can be expressed as: 


$$
T R=\frac{T_{\max }-T_{\min }}{T_{\max }} \quad[p u]
$$

where $T_{\max }$ and $T_{\min }$ are the maximum and minimum values of the instantaneous torque.

Since copper losses depend on current density $(\delta)$ [15], iron losses and power factor depend on stator flux density $\left(B_{s}\right)$ [16], and torque ripples depend on the stator and rotor pole arcs $(\alpha, \beta)[17]$, the design vector has been selected to contain the following effective parameters:

$$
X=\left\{\begin{array}{lllllll}
D & l & \text { sr } & \alpha & \beta & \delta & B_{s}
\end{array}\right\}
$$

\subsection{Fuzzy Aggregation Of Objectives}

The main steps of fuzzy aggregation process are:

\subsubsection{Objective identification}

The first step consists of defining all objectives of the problem in terms of fuzzy sets. The shape of the membership function most suitable for the problem should be selected [18].

\subsubsection{Fuzzification}

Fuzzification means that the calculated objectives are transformed into fuzzy quantities, which are also referred to as linguistic values. This means that for each objective function $f$, there is value of $\mu$ gives the degree of fulfilment or satisfaction of this objective.

In our application, the following simple two memberships can be used:

- Efficiency and power factor membership function (S-shaped):

$$
\mu_{i}(X)=\left\{\begin{array}{cc}
1 & f_{i}(X) \geq M_{i} \\
1-2\left[\frac{M_{i}-f_{i}(X)}{M_{i}-m_{i}}\right]^{2} & 0.5\left(m_{i}+M_{i}\right)<f_{i}(X)<M_{i} \\
2\left[\frac{f_{i}(X)-m_{i}}{M_{i}-m_{i}}\right]^{2} & m_{i}<f_{i}(X)<0.5\left(m_{i}+M_{i}\right) \\
0 & f_{i}(X) \leq m_{i}
\end{array}\right.
$$

- Volume and torque ripple membership function (Z-shaped):

$$
\mu_{i}(X)=\left\{\begin{array}{cc}
1 & f_{i}(X) \leq M_{i} \\
1-2\left[\frac{f_{i}(X)-M_{i}}{M_{i}-m_{i}}\right]^{2} & 0.5\left(m_{i}+M_{i}\right)>f_{i}(X)>M_{i} \\
2\left[\frac{m_{i}-f_{i}(X)}{M_{i}-m_{i}}\right]^{2} & m_{i}>f_{i}(X)>0.5\left(m_{i}+M_{i}\right) \\
0 & f_{i}(X) \geq m_{i}
\end{array}\right.
$$

where $M_{i}$ is the function value corresponding to the highest degree of acceptance and $m_{i}$ is the function value corresponding to minimum or zero degree of satisfaction. For the efficiency and power factor, the maximum values $M_{i}$ can be selected from the graph shown in Figure 1 . The graph is just an example obtained from many previously published designs [15]. 


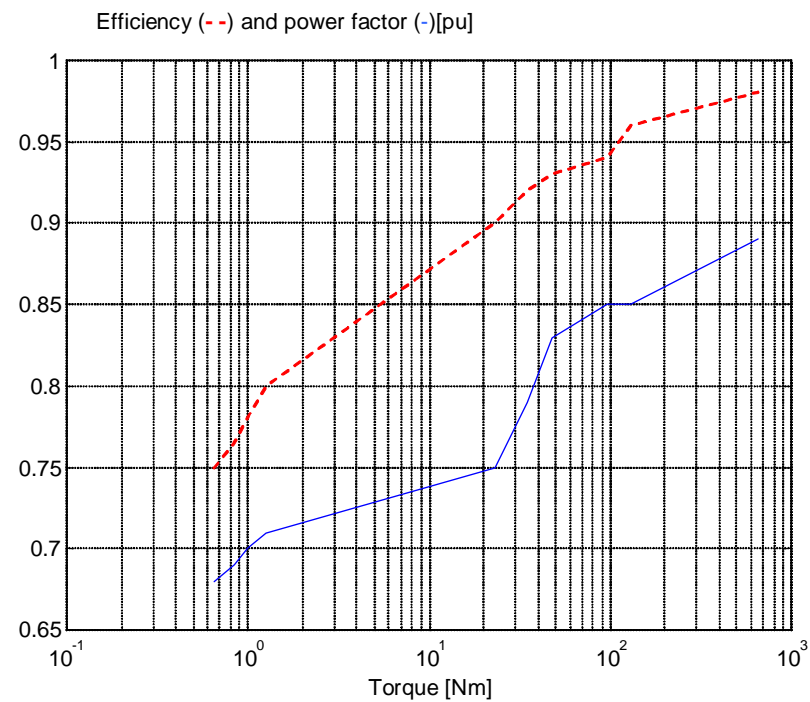

Figure 1 Example of maximum values for efficiency and power factor.

\subsubsection{Fuzzy Combination}

This allows comparing and combining different degrees of satisfaction in a global one. The degree of acceptance of each configuration or design is defined as the intersection of all the single objective membership functions. The optimisation procedure therefore must look for a configuration, which maximises the global degree of satisfaction as:

$$
\max _{X} \operatorname{mize}_{\mathrm{x}}\left\{\mu_{t}\right\}
$$

where $\mu_{t}$ is the global degree of satisfaction and can be defined as:

$$
\mu_{t}=\mu_{1} \cap \mu_{2} \cap \mu_{3} \ldots \ldots \ldots \ldots . . . . \cap \mu_{k} \quad \text { for } k \text { objective functions }
$$

or $\max _{x}\left\{\prod \mu_{j}\right\} \quad j=1,2, \ldots \ldots \ldots \ldots . ., k$

A flow chart of the fuzzy aggregation method is clear in Figure 2

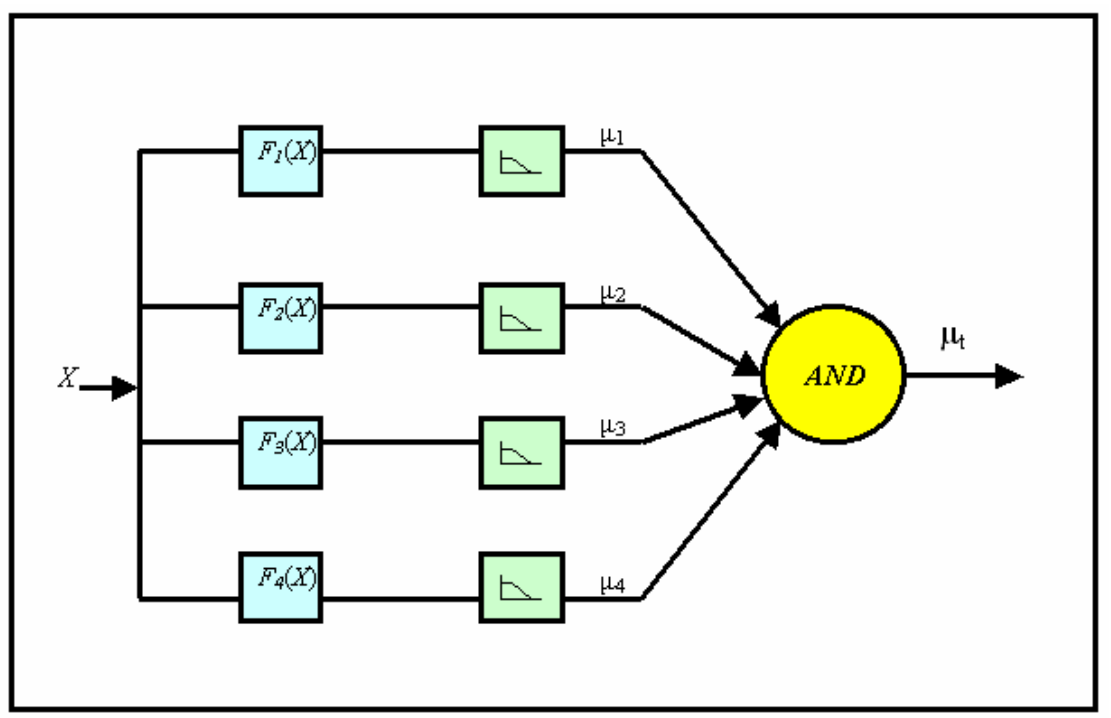

Figure 2 Flow chart of Fuzzy Optimisation 


\subsection{MOTOR MODEELING}

\subsubsection{Static Model}

i- Magnetisation curves

The model adopted in this paper is a modified version of the analytical model that published in reference [19]. This model connects the fundamental design of an SRM to a simple analytical expression, which summarises the terminal magnetisation characteristics. The modelling procedure takes simple piecewise linear models which are based on the geometry and turns per phase of the SRM and maps them through simple and fast algorithms into the following analytical expression:

$$
\lambda(i, \vartheta)=a_{1}(\vartheta)\left[1-e^{\left(a_{2}(\vartheta) \cdot i\right)}\right]+a_{3}(\vartheta) \cdot i
$$

where $a_{1}, a_{2}$, and $a_{3}$ are the magnetisation coefficients, which are functions of rotor position. These coefficients are approximated at aligned and unaligned positions in reference [19] but it was found that these initial values are most suitable for heavily saturated motors and not very good for optimisation purposes. So a simple direct optimisation algorithm based on Simplex optimisation is adopted to find the most suitable coefficients for the model to best fit the FEA solutions at the aligned and unaligned positions. This hybrid FEA-Analytical technique comprises some of the FEA accuracy with the simplicity of the analytical model and still less time consuming compared to full FEA simulation[15].

ii- Static Co-energy and Torque curves

To compute the static torque curves $T(\theta, i)$, the function or table of co-energy values $W(\theta, i)$ is required. From equation (12), the co-energy can be computed as:

$$
\begin{aligned}
& W(\theta, i)=\int_{0}^{i} \lambda(\theta, i) d i \\
& W(\theta, i)=a_{1}(\theta) i+\frac{a_{1}(\theta)}{a_{2}(\theta)}\left(1-e^{\left(a_{2}(\theta) i\right)}\right)+\frac{a_{3}(\theta)}{2} i^{2}
\end{aligned}
$$

The static torque can therefore be computed numerically as:

$$
T(\theta, i)=\frac{\partial W(\theta, i)}{\partial \theta} \text { for constant } i \text {. }
$$

\subsubsection{Steady state dynamic model}

For accurate simulation of the steady state performance, the actual current waveform for normal conditions must be represented as accurate as possible. Current prediction requires the definition of the magnetic behaviour of the motor in the form of $i(\vartheta, \lambda)$ and the solution of the differential circuit equations.

The phase equation of the motor has the following formula:

$$
\frac{d \lambda(\theta, i)}{d t}=V-R i
$$

where $\mathrm{R}$ is the per-phase resistance and $\mathrm{V}$ is the applied voltage.

The applied voltage can be expressed as:

$$
V=\left\{\begin{array}{lr}
V_{s} & \theta_{O}<\theta<\theta_{C} \\
-V_{S} & \theta_{C} \leq \theta<\theta_{q} \\
0.0 & \theta \geq \theta_{q}
\end{array}\right.
$$

where $V_{s}$ is the dc supply voltage, $\theta_{O}$ is the turn-on angle, $\theta_{C}$ is the turn-off or commutation angle, and $\theta_{q}$ is the quenching angle. 
Under normal operating conditions, the speed is constant and hence the equation (16) can be expressed as:

$$
\frac{d \lambda(\theta, i)}{d \theta}=\frac{1}{\omega}(V-R i)
$$

To obtain the current waveforms, the following steps have to be undertaken:

(i) Equation (18) is integrated numerically for one phase; the other phases are the same but phase-shifted by a step angle $\alpha_{s}$.

(ii) After each step in the integration, the current is updated according to the new value of the flux linkage.

(iii) The new value of current is used in the next integration step and so on.

These steps can be summarised as:

$$
\theta_{k}=\theta_{k-1}+\Delta \theta
$$

And

$$
\lambda_{k}=\frac{1}{\omega}\left(V-R i_{k-1}\right) \Delta \theta+\lambda_{k-1}
$$

where $\mathrm{k}$ is the number of the integration step and $\Delta \theta$ is the integration step angle.

The steady state dynamic torque, co-energy, and magnetisation curves can be obtained from the static characteristics by interpolation following the estimated current / angle waveform. From theses torque curves the resultant torque can be estimated.

\subsubsection{Losses Calculation}

-The mechanical losses due to friction in the bearings and windage between stator and rotor can be approximated by[20]:

$$
\Delta P_{m}=17 . n_{r} \cdot D^{2} L
$$

where $n_{r}$ is the motor speed [rpm].

-Electrical copper loss can be computed as:

$$
\Delta P_{c u}=q \cdot I_{m s}^{2} \cdot R
$$

where R is the phase resistance and $I_{m s}$ is the effective phase current.

- For iron losses estimation there are many models however for fast and still accurate estimation the model of reference [21] has been adopted. The model is based on experimental work and background knowledge of how core losses vary in conventional machines:

$$
\begin{aligned}
\Delta_{c} & =a f_{s}^{b}\left(\frac{V}{f_{s}}\right)^{c} \\
\Delta P_{c} & =\sigma_{S} V_{S T} \Delta_{C}
\end{aligned}
$$

where $\mathrm{V}$ is the effective phase voltage [22] or the peak supply voltage [21], $f_{s}$ is the supply frequency, $\sigma_{s}$ is the steel mass density, $a, b$, and $c$ are constants, and $V_{S T}$ is the total motor steel volume.

\subsection{Constraint Functions}

i- Constraints On Motor Proportion And Physical Dimensions:

- Stator Pole Width to Pitch Ratio $r_{1}$ :

For self-starting motor, the stator pole width must not be less than the step angle i.e.

$$
r_{1} \geq \frac{2}{n}
$$


where $r_{1}$ is the stator pole width to pitch ratio or normalised stator pole width and $\mathrm{n}$ is the number of rotor poles.

\section{-Rotor Pole Width to Pitch Ratio $r_{2}$ :}

$$
r_{2} \geq r_{1} \cdot \frac{n}{m}
$$

where $\mathrm{m}$ is the number of stator poles. This constraint is placed to ensure that the rotor pole width is greater than or equal to the stator pole width and hence assures self-starting of the motor with adequate time for the current to decrease in each stroke.

-Motor Aspect Ratio $(A R=L / D)$ :

$$
0.3 \leq A R \leq 3.0
$$

This constraint ensures a sensible aspect ratio for the motor.

\section{-Angular Clearance Constraint:}

The stator pole arc $\alpha$ must be less than the rotor interpolar arc $\beta$ to ensure angular clearance between the stator and rotor pole corners and hence ensure sufficiently low unaligned inductance $L_{u}$

$$
\alpha<\beta^{\prime}
$$

-Slot Filling Factor $F f$ :

$$
f f \leq f f_{m}
$$

where $\mathrm{ff}_{\mathrm{m}}$ is the maximum slots filling factor which depends on the type of coil and amount of insulation. The value equals 0.4 for dropped in coils and 0.7 for pushed through coils. -Airgap Length lg:

$$
l_{g} \geq 0.2 \mathrm{~mm}
$$

This constraint ensures that the airgap is mechanically acceptable.

-Positive Value Constraints:

$$
X_{i}>0
$$

where $\mathrm{X}_{\mathrm{i}}$ is any of the optimisation variables

ii- Constraints on Magnetic and Electric Loading:

-Stator Current Density $\delta$ :

$$
\delta \leq \delta_{\max }
$$

where $\delta_{\max }$ is the maximum allowable current density. The effective current density ranges from 3 to 6 for totally enclosed natural cooling motors and from 7 to 10 for external selfventilation motor [3].

-Stator Tooth Flux Density $B_{s}$ :

$$
B_{s} \leq B_{s t}
$$

where $B_{s t}$ is the saturating flux density, which ranges from 1.7 to 2.2 Tesla, depending on the steel material.

\section{iii- Performance Constraints:}

-Steady State Average Torque $T_{a}$ :

$$
T_{a} \geq k T_{o}
$$

Where $T_{o}$ is the full load output torque and $k$ is the overload factor (usually 1.15). 
-Efficiency $\eta$ :

$$
\eta_{\min } \leq \eta \leq \eta_{\max }
$$

where $\eta_{\min }$ and $\eta_{\max }$ are the minimum and maximum allowed efficiencies. This constraint ensures practical efficiency of the motor and hence suitable temperature rise.

- Generalised Power Factor PF :

$$
P F_{\text {min }} \leq P F \leq P F_{\text {max }}
$$

where $P F_{\min }$ and $P F_{\max }$ are the minimum and maximum allowed power factors. This constraint ensures suitable $\mathrm{KVA} / \mathrm{KW}$ requirements of SRM drive where increasing the $P F_{m}$ tends to lower the volt-ampere requirements of the drive.

iv- Search space constraints:

$$
\begin{gathered}
0.05 \leq D \leq 0.5 \\
0.05 \leq L \leq 0.5 \\
0.55 \leq s r \leq 0.63
\end{gathered}
$$

These constraints are necessary to limit the search space into a practical domain.

It is clear that there are a number of variables and constraints, which are, involved directly in the optimisation process such as number of stator and rotor poles, Stator Back depth to stator pole width ratio, winding areas, and shaft diameter. For this reason the design and analysis program is executed at each optimisation step to complete the design data and compute the objective and constraint functions.

\subsection{Fitness Function}

The design optimisation procedure can be implemented by converting the constrained problem into an unconstrained one using exterior penalty functions. This ensures that the constraints are satisfied during the optimisation process [3, 7, 9, 23].

Using a static penalty factor, the fitness or augmented function can be written as:

$$
f(X)=F(X)+r \sum_{j=1}^{m_{i}} W_{j} \quad\left\{\max \left(0, g_{j}(X)\right\}^{2}\right.
$$

And the standard form of $g_{i}(X)$ is:

$$
g_{j}(X) \leq 0
$$

where $r$ is the static penalty factor, $W_{j}$ is weighting factor and $g_{j}(X)$ is the inequality constraint functions. The best value for the static penalty factor $r$ is problem dependent and can be found only through trial and error $[3,7,9]$.

\section{RESULTS AND CONCLUSION}

The proposed optimisation algorithm as well as the simplex optimisation method has been applied to a $40 \mathrm{~kW} / 200 \mathrm{Nm}, 240 \mathrm{~V}$, 6/4, 3phase, $2000 \mathrm{rpm}$, air-cooled SRM. The main dimensions of the starting design have been taken from reference [24]. The motor is then resimulated using steel M22 at $B_{s}=2 T$ and taken as a good initial point. The problem is solved with seven variables and fifteen active constraints [equations (25): (39)]. The airgap is assumed constant at $0.947 \mathrm{~mm}$ during optimisation process to achieve fair comparison with the initial design. The proposed values of $M_{i}$ and $m_{i}$ for the outer volume, power factor, efficiency and torque ripples are selected respectively as: 


$$
\begin{aligned}
& M_{i}=\left[\begin{array}{llll}
0.0017 & 0.850 & 0.98 & 0.1996
\end{array}\right] \\
& m_{i}=\left[\begin{array}{llll}
0.00853 & 0.550 & 0.88 & 1.1974
\end{array}\right]
\end{aligned}
$$

The main results of the optimised design and simulation are explained in Table1 and in figures 3:8.

\begin{tabular}{|c|c|c|c|c|}
\hline \multirow[t]{2}{*}{ Main parameters } & \multirow{2}{*}{$\begin{array}{l}\text { Initial } \\
\text { design }\end{array}$} & \multirow{2}{*}{$\begin{array}{l}\text { Fuzzy- } \\
\text { Simplex }\end{array}$} & \multicolumn{2}{|c|}{ Fuzzy } \\
\hline & & & Genetic & $\begin{array}{l}\text { Genetic- } \\
\text { Simplex }\end{array}$ \\
\hline Population Size & - & - & 3000 & 3000 \\
\hline Number of crossovers & - & - & 750 & 750 \\
\hline Number of mutations & - & - & 60 & 60 \\
\hline Number of generations & - & - & 50 & 50 \\
\hline Rotor outer diameter & 0.18972 & 0.1700 & 0.1852 & 0.1644 \\
\hline Axial stack length & 0.18972 & 0.1819 & 0.1611 & 0.1809 \\
\hline Split ratio & 0.55 & 0.53796 & 0.5480 & 0.5288 \\
\hline Stator pole arc [degrees] and [pu] & $30 / 0.5$ & $30.29 / 0.505$ & $31.15 / 0.519$ & $30.74 / 0.5123$ \\
\hline Rotor pole arc [degrees] and [pu] & $36 / 0.4$ & $39.07 / 0.434$ & $48.04 / 0.533$ & $46.28 / 0.5143$ \\
\hline Current density $\left[\mathrm{A} / \mathrm{mm}^{2}\right]$ & 4.4616 & 4.855 & 4.3737 & 4.6671 \\
\hline Maximum stator flux density [T] & 2 & 2.0552 & 2.0457 & 2.053 \\
\hline Average torque $[\mathrm{Nm}]$ & 178.17 & 208.1185 & 205.779 & 203.6907 \\
\hline Outer Volume (estimated) $\left[\mathrm{m}^{3}\right]$ & 0.0271 & 0.021326 & 0.02324 & 0.020339 \\
\hline Power factor (estimated) & 0.745 & 0.7983 & 0.7997 & 0.7924 \\
\hline Efficiency (estimated) \% & 94.3194 & 95.1427 & 94.9386 & 94.7644 \\
\hline Torque ripples \% (estimated) & 79.83 & 64.873 & 33.095 & 36.331 \\
\hline Total degree of acceptance [pu] & 0.088 & 0.3927 & 0.55419 & 0.6060 \\
\hline
\end{tabular}

Table 1 Main Parameters of the SRM design

The results show the following

- Improvement in all objective functions with respect to the initial design especially for torque ripples, however it is fair to say that the initial design was intended for wide speed applications rather than low torque ripple applications.

- Fuzzy-simplex optimisation algorithm achieved slightly better efficiency and power factor and inferior outer volume and torque ripples with respect to the final solution, which assures the existence of conflict among objective functions.

- The proposed optimisation technique (FGS) achieved the highest degree of acceptance and improved than that of fuzzy-simplex and fuzzy-genetic algorithm by approximately $21 \%$ and $5 \%$ respectively.

- Coupling of both simplex and genetic algorithms improves the slow finishing of genetic algorithm and eliminates the local minimum sticking of simplex algorithm.

- $\quad$ The existence of fuzzy aggregation eliminates the continual existence of decision maker.

- $\quad$ The time stepping finite element analysis (FEA) of the motor has been done as shown in figures 4:7. These figures showed good accuracy of the adopted model and improvement in the torque ripple from $36.33 \%$ to $32.7 \%$ and a slight decrease in average torque $\sim 4 \%$.

- The genetic algorithm is faster in determining the interval of optimum design than determining the optimum design itself and this is clear in Figure 3. 


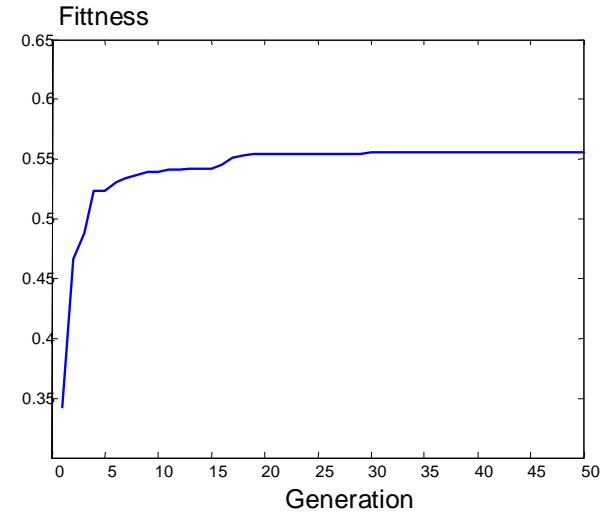

Figure 3 Trace of best fitness Function

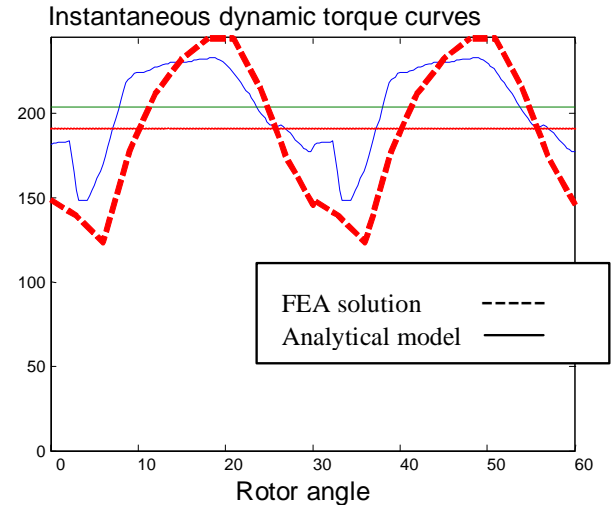

Figure 4 Average and resultant torque

curves.

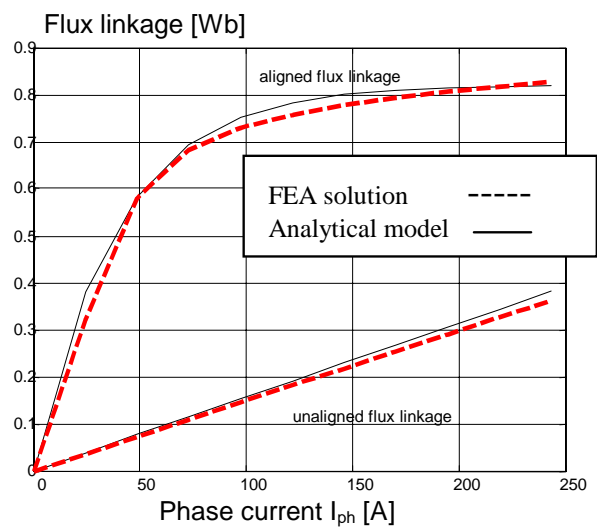

Figure 5 Aligned and unaligned magnetisation curves.

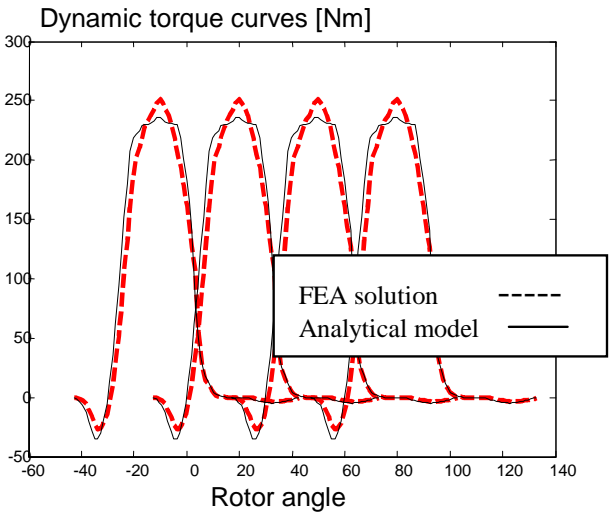

Figure 6 Dynamic torque curves

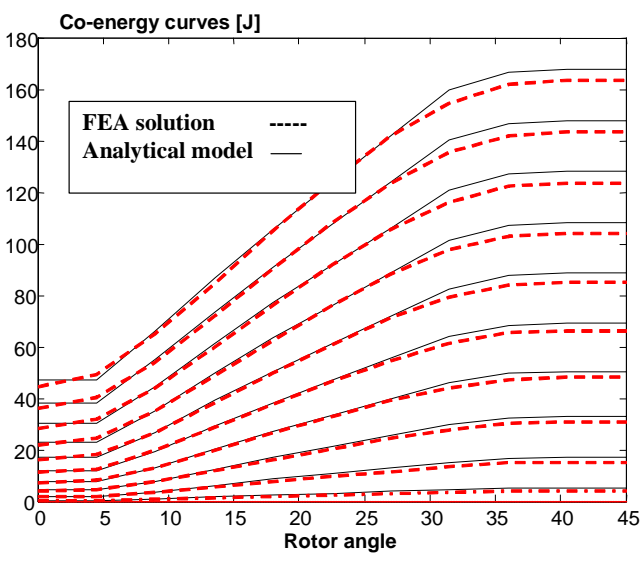

Figure 7 Co-energy Curves

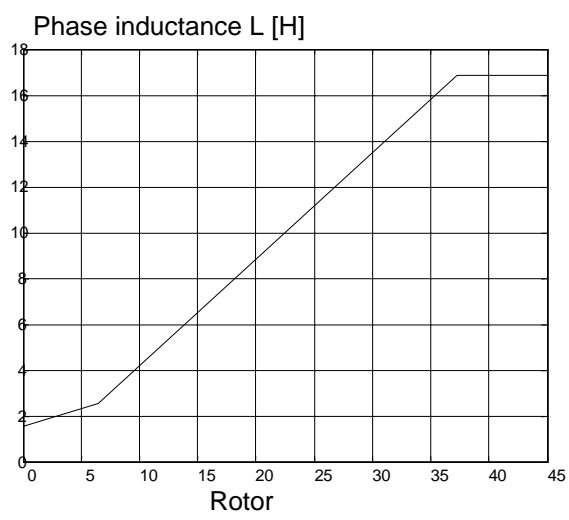

Figure 8 Inductance Profile

\section{REFERENCES}


[1] C. A. C. Coello, "An Empirical Study of evolutionary Techniques for multiobjective optimization in engineering design, $\mathrm{PhD}$ thesis," Dept. of Computer science, Tulane University, 1996.

[2] D. Cvetkovic and I. Parmee, "Evolutionary Design and Multi-objective Optimisation," presented at '6th European Congress on Intelligent Techniques and soft computing EUFIT'98, Aachen, Germany, 1998.

[3] A. El-Wakeel and A. C. Smith, "Optimal Design Of Switched Reluctance Motors Using Genetic Algorithms," presented at Proceedings of ICEM 2002, International Conference on Electrical Machines, Bruges, Belgium, 26 - 28 Aug., 2002.

[4] E. Zizter, "Evolutionary Algorithms for Multiobjective optimisation: Methods and Applications, PhD thesis." Zurich: Swiss Federal of Technology, 1999.

[5] E. Zitzler, "Evolutionary Algorithms For Multiobjective Optimization," presented at Evolutionary Methods For Design Optimisation And Control, CIMNE, Barcelona, Spain, 2002.

[6] N. Binichi and S. Bolongnani, "Design Optimisation of Electric Motors by Genetic Algoritghms," IEE Proc., Electr. Power Appl., vol. 145, pp. 475-483, Septemper 1998.

[7] A. El-Wakeel and A. C. Smith, "GENETIC DESIGN OF A SWITCHED RELUCTANCE MOTOR BASED ON MINIMUM OUTER VOLUME," presented at Proceedings of ICEENG 2002, the 3rd International Conference on Electrical Engineering, Cairo, Egypt, 14 - 16 May 2002.

[8] S. Palko and T. Jokinen, "Optimisation of squirrel cage induction motors using finite element method and genetic algorithms," Proceedings of IEE conference EMD97, pp. 21-25, 1-3 Sept. 1997.

[9] H. B. Ertan and M. Tohumcu, "A method for optimum design of switched reluctance machines," presented at Proceedings of ICEM '88, International Conference on Electrical Machines, Pisa, Italy, 12 - 14 September, 1988.

[10] A. C. Smith, A. S. El-Wakeel, and A. Wallace, "Formal design optimization of PM drive couplings," IEEE, 2002.

[11] A. El-Wakeel, "Design Optimisation For Fault Tolerant Switched Reluctance Motors, PhD thesis," in Dept. of Electrical Engineering and Electronics. Manchester: Umist, 2003.

[12] A. El-Wakeel, "DESIGN OPTIMISATION OF ELECTRIC MACHINES," presented at Proceedings of the 4th ICEENG Conference, 23-25 Nov. 2004, 2004.

[13] T.-K. C. Yong-Hwan OH, Min-Kyu Kim, and Hyun-Kyo Jung, "Optimal Design of Electric Machines Using Genetic Algorithms Coupled with Direct Method," IEEE Transactions on Magnetics, vol. 35, pp. 1742-1745, 1999.

[14] T. J. E. Miller, "Converter Volt-Ampere Requirements of Switched Reluctance Motor Drive," IEEE Trans. on Ind. Appl., vol. IA-21, pp. 1136 - 1144, September/October 1985.

[15] A. El-Wakeel, "Design Optimisation For Fault tolerant Switched Reluctance Motors," in Electrical Engineering and Electronics. Manchester: Umist, 2003, pp. 256.

[16] R. Krishnan, R. Arumugam, and J. F. Lindsay, "Design procedure for switched reluctance motors," presented at Conference Record of IAS '86, the 1986 IEEE Industry Applications Society 21st Annual Meeting, Denver, CO, USA, 28 September - 3 October, 1986.

[17] F. Sahin, H. B. Ertan, and K. Leblebiciogula, "Optimum Geometry for Torque Ripple Minimization of Switched Reluctance Motors," IEEE Transactions on Energy Conversion, vol. 15, pp. 30-39, March 2000. 
[18] M. Chiampi, C. Ragusa, and M. Repetto, "Fuzzy Approach for multiobjective optimization in Magnetics," IEEE Transaction on Magnetics, vol. 32, pp. 1234-1237, May 1996.

[19] D. A. Torrey, X.-M. Niu, and E. J. Unkauf, "Analytical Modelling of Variable Reluctance Machine Magnetisation Characteristics," IEE Proc., Electric power Application., vol. 142, pp. 14 - 22, January 1995.

[20] E. Levi, Polyophase Motors: A Direct Approach to Their Design: John Wiley \& Sons, 1984.

[21] A. R. Miles, "Design of a 5MW, 9000V switched reluctance motor," IEEE Transactions on Energy Conversion, vol. 6, pp. 484 - 491, September, 1991.

[22] H. M. Metwally, J. Faiz, and J. W. Finch, "Core loss in switched reluctance motors experimental results," presented at Proceedings of ICEM '88, International Conference on Electrical Machines, Pisa, Italy, 12 - 14 September 1988.

[23] M. Ramamoorty, Computer-Aided design of electrical equipment: Published by Ellis Horwood Limited, 1988.

[24] B. F. K.M Rahman, G. Suresh, A.V. Rajarathnam, and M. Ehsani, "Advantages of Switched Reluctance Motor Applications to EV and HEV: Design and control Issues," IEEE Transactions on Industry Applications, vol. 36, pp. 111-121, January/February, 2000. 